Revista Electrónica Complutense de Investigación en Educación Musical ISSN-e: 1698-7454

http://dx.doi.org/10.5209/RECIEM.53371

\title{
Aplicaciones didácticas del lenguaje Soundpainting en diferentes ámbitos educativos: una herramienta para la creación en tiempo real
}

\author{
Òscar Vidal Belda ${ }^{1}$; Remigi Morant Navasquillo
}

Recibido 1 Mayo de 2016 / Aceptado 22 Junio de 207

Resumen. El objetivo de esta investigación ha sido analizar y describir cómo se ha aplicado el lenguaje Soundpainting en tres ámbitos educativos diferentes. Partiendo de la metodología cualitativa se seleccionaron un conservatorio, un centro de educación primaria y una escuela de música como estudio de caso colectivo. Las diferentes sesiones fueron grabadas en vídeo, revisadas y codificadas con la ayuda de programas de análisis cualitativo. Posteriormente se realizó una entrevista semiestructurada a cada uno de los profesores. Los resultados muestran que aplicando el lenguaje Soundpainting desde la perspectiva del juego y haciendo un uso didáctico de los gestos se facilitó el desarrollo de las capacidades musicales y personales en los alumnos de los tres ámbitos educativos observados, tal y como se describe en las conclusiones.

Palabras clave: creación en tiempo real; soundpainting; aplicaciones didácticas.

\section{[en] Didactic application of the Soundpainting language in different} educational fields: a tool for music creation in real time

\begin{abstract}
The goal of this research has been to analyze and describe how this language is being used in three different educational frameworks. According to qualitative methodology a conservatory, a primary school and a music school were selected as a collective case study. The different sessions were recorded, revised and codified using different software of qualitative data analysis. Subsequently each teacher was invited to a semi-structured interview. Results show that applying Soundpainting in a playful way and using gestures as a didactic tool made it easier the development of personal and musical abilities of the students in three different educational environments, as described in the final conclusions.
\end{abstract}

Keywords: Real time creation; Soundpainting; didactical applications.

Sumario. 1. Introducción. 2. Fundamentación teórica. 2.1. Diferentes sistemas para la creación musical en tiempo real. 2.2. Creación sonora en tiempo real en el aula. 2.3. Estudios actuales sobre Soundpainting. 2.4. Soundpainting en el entorno educativo. 3. Metodología de la investigación. 3.1. Diseño de la investigación. 3.2. Técnicas e instrumentos de recogida de datos. 3.3. La muestra. 3.4. Procedimiento de análisis de los datos. 4. Análisis y discusión de los resultados. 4.1. Primer caso: Conservatorio de Privas. 4.2. Segundo caso: Escuela de Música de Tres Cantos. 4.3. Tercer caso: Jossefiau Primary School, Salzburg. 4.4. Discusión de Resultados. 5. Conclusiones. 6. Líneas de investigación futuras. 7. Referencias bibliográficas.

\footnotetext{
$1 \quad$ IES Benlliure (Valencia)

E-mail: ovidal@iesbenlliure.es

2 Universitat de València

E-mail: remigi.morant@uv.es
} 
Cómo citar: Vidal Belda, Ò.; Morant Navasquillo, R. (2017). Aplicaciones didácticas del lenguaje Soundpainting en diferentes ámbitos educativos: una herramienta para la creación en tiempo real, en Revista Electrónica Complutense de Investigación en Educación Musical, 15, 329-350.

\section{Introducción}

En los inicios del curso escolar 2005-2006 empezamos a interesarnos por la improvisación libre y por la creación musical en tiempo real; un término que ya antes habíamos leído y que se relacionaba con la música electroacústica en directo. Ciertas informaciones sobre algunos sistemas de composición en tiempo real sirvieron de fuente para ir extrayendo ideas, ambientes, colores y, lo más importante gestos para poder comunicarse con los alumnos y hacer creaciones musicales en el aula; algunos de estos signos pertenecientes al sistema Conducting $\square$ de L. D. "Butch" Morris, o ideas de usar colores como medio de comunicación extraídas de la obra "Cobra" de John Zorn, e incluso algunos gestos inventados en el aula conforme nos hicieron falta.

Todo este trabajo se tradujo en la participación, en febrero de 2006, con nuestros alumnos en el festival de música experimental NITS d'AIELO i ART en el cual hicimos una pequeña creación sonora. De nuestra actuación en este festival surgió la oportunidad de realizar un concierto en Barcelona con Walter Thompson, creador del lenguaje Soundpainting. Fueron los propios alumnos los que, al finalizar nuestra actuación con Thompson, se interesaron en utilizar este lenguaje de signos en el aula.

Soundpainting es un lenguaje de signos creado en los años 80 por el músico de Nueva York Walter Thompson para la creación de arte sonoro en tiempo real conocido como The Art of Live Composition (Thompson, 2006). Este lenguaje surgió de la necesidad de Thompson de darle forma a la creación sonora al tiempo que se está ejecutando. El Soundpainter es un compositor que usa los signos como herramienta de creación que, dibujándolos en el espacio, se convertirán en sonidos, su materia prima para la creación musical. La composición con este lenguaje combina la aleatoriedad con otras indicaciones exactas que contienen información de tipo sintáctico sobre quién, qué, cómo y cuándo deben producirse los sonidos por parte de los intérpretes; es lo que Thompson (2006) denomina Soundpainting Syntax. Una composición Soundpainting es el resultado de la comunicación entre el Soundpainter y las respuestas sonoras y visuales de los intérpretes en un proceso vivo e interactivo. Aunque Soundpainting surgió en los cursos de verano de Woodstock (New York, USA) en 1974, no fue hasta la década de los 90 cuando empezó a desarrollarse en el ámbito educativo.

Una de las inquietudes de los docentes ha sido desde siempre ofrecer recursos para expresarse a través de la creación y la interpretación, fomentando así la participación activa en el fenómeno musical desde la diversidad colectiva del aula. De la propia experiencia en el uso educativo de este lenguaje surge el interés y el objetivo de esta investigación: analizar cómo se está aplicando Soundpainting en diferentes ámbitos educativos, así como los interrogantes que se plantean basándose en las propias inquietudes y curiosidades. El estudio se ha realizado observando, analizando y describiendo cómo se aplica este lenguaje en tres ámbitos educativos diferentes 
(Conservatorio, Escuela de Música y Centro de Educación Primaria) para realizar la posterior triangulación de los datos que se han obtenido en cada uno de los tres casos, enmarcando la investigación en el campo de "La música como investigación, investigaciones relativas a herramientas y proyectos relacionados con la música" (Díaz, 2006, p. 104).

La elección de Soundpainting como objeto de estudio parte de la experiencia de aplicar este nuevo lenguaje como recurso para la composición musical en la etapa de educación secundaria, al observar su potencial para desarrollar la creación musical así como diversas capacidades musicales en el alumnado.

Bajo el título Aplicaciones didácticas del lenguaje Soundpainting en diferentes ámbitos educativos: una herramienta para la creación en tiempo real hay un interés por saber además de cómo se utililiza Soundpainting y qué aplicación didáctica se le da. Para ello planteamos el siguiente objetivo principal:

Analizar cómo se está utilizando el lenguaje Soundpainting en diferentes ámbitos educativos y con qué finalidades.

Para alcanzar nuestro objetivo nos hicimos las siguientes preguntas:

- ¿Qué diferencias o similitudes existen en la aplicación didáctica de Soundpainting en diferentes ámbitos educativos?

- ¿Qué tipo de capacidades se desarrollan en el uso didáctico de Soundpainting?

- ¿De qué manera se favorece el desarrollo de capacidades en los alumnos con la aplicación (o el uso) de Soundpainting?

\section{Fundamentación teórica}

Antes de entrar en el análisis del estado de la cuestión, y dado que Soundpainting es definido por Walter Thompson (creador del lenguaje) como el arte de la composición en vivo, creemos necesario hacer una primera exploración del término creación (composición) en tiempo real (en vivo). El término "en tiempo real" ha sido usado frecuentemente en el terreno de la informática y alude, a lo que Norro (2006) define como sincronismo causal, es decir, la capacidad de ciertos dispositivos tecnológicos de poder procesar o modificar una señal de audio, vídeo o imagen al mismo tiempo que ésta se produce, siendo la segunda causa de la primera. A partir del avance de la tecnología desarrollado en los años setenta en el terreno musical, el término se fue generalizando (Norro, 2006), y por ende ha sido utilizado de manera más o menos indiscriminada en el ámbito musical. Así pues, esta generalización y el uso indiscriminado del término composición en tiempo real (composición improvisada, improvisación dirigida, improvisación libre, creación instantánea) nos sitúa en una posición concreta. Hay creación en la improvisación e improvisación (en su sentido creativo) en la composición, pero no son conceptos exactamente iguales (Molina, 2008). Nosotros entendemos la composición como un acto creativo que puede realizarse en tiempo real o en tiempo diferido. Creemos oportuno adoptar esta posición respecto a estos dos términos, ya que Soundpainting establece en su código de gestos la improvisación como un elemento de respuesta entre el intérprete y el Soundpainter. 
En conclusión, Norro (2006, p. 112) define la composición en tiempo real como "una sucesión de sonidos dispuestos en determinado orden, cuya estructura, a su vez, emerge de un acto mental, suscitando una idea de conjunto que los contiene, que es elaborada, interpretada y registrada en forma simultánea en un mismo lapso temporal".

\subsection{Diferentes sistemas para la creación musical en tiempo real}

En relación a diferentes softwares que reflejen en música el movimiento u otras manifestaciones artísticas visuales hemos encontrado algunas investigaciones en referencia a esta dimensión de la composición en tiempo real relacionada con los medios electrónicos. Uno de los más interesantes es el de Solís (2006) Improvisatory Music and Painting Interface (IMPI). Se trata de un software para la creación de improvisaciones audiovisuales interpretadas en tiempo real realizadas en grupo, con el objetivo de dar forma a improvisaciones colectivas libres. La finalidad es obtener productos musicales de calidad usando un lenguaje gráfico. Uno de los puntos fuertes del sistema IMPI es la integración de material sonoro preparado con anterioridad dentro del campo de la improvisación, elemento éste que comparte con algunos gestos de Soundpainting. Así pues, el autor analiza, desde un punto de vista estético, el concepto de improvisación guiada o controlada tal y como él expresa: "controlled improvisation".

En esta línea se sitúa el estudio de Dessen (2010) New Polyphonies: Score Streams, Improvisation and Telepresence, que presenta un método de composición en el cual la notación va apareciendo también en la pantalla de un ordenador y va siendo interpretada por un grupo de improvisadores. El estudio se fundamenta en métodos basados en la teoría del compositor-improvisador entendidos como acciones colaborativas de creación en tiempo real.

The Mad Scientist Machine, de Smulovitz (2010) es un sistema que permite a un usuario (director/compositor) integrar a un grupo de intérpretes en una interpretación de improvisación estructurada. El usuario (remoto o local) puede utilizar el software para controlar un Led situado frente a cada intérprete. Cada color del Led indica una instrucción de interpretación diferente. Así pues, señales blancas indican tonos largos, o marrones indican realizar ruido, por ejemplo.

Hay que esperar a Lawrence D. "Butch" Morris para encontrar un sistema de creación en vivo basado únicamente en un código de signos, o lo que él denominó y registró como "Conducting" y que Stanley (2009) estudia en su tesis doctoral Butch Morris and the art of conduction. Aunque en este estudio aparece algún vestigio del uso de este sistema creado por Morris en el ámbito educativo, desde su creación ha estado exclusivamente vinculado al ámbito profesional de lo performativo.

El caso de "Cobra" (1984) de John Zorn, para el cual creó un sistema de signos codificados que utiliza en tiempo real mostrándolos a los integrantes del grupo en diferentes cartulinas, aunque usado inicialmente en el ámbito profesional, también se ha utilizado ocasionalmente en el ámbito educativo. 


\subsection{Creación sonora en tiempo real en el aula}

Componer en tiempo real en grupo es configurar una narrativa sonora al momento, nutrida por la aportación y la dote emocional de los participantes. (Espinosa, 2005, p. 88)

Existen muchas investigaciones que ponen de manifiesto la importancia de la creación musical en nuestras aulas (Cárdenas, 2003; Díaz, 2006; Rusinek, 2005), aunque son bien pocos los estudios relacionados con la creación musical en tiempo real en el ámbito educativo, y los pocos que existen se han centrado en la educación secundaria.

En muchos de los casos, lo que se hizo en el pasado fueron intentos de crear espontáneamente música en el aula partiendo de un set de signos básicos inventados en el momento por el docente y que servían a los fines propios de una actividad concreta:

De modo que no se preocupen en cuanto a qué notas van a tocar; yo les indicaré la altura general del sonido -si es grave, medio o agudo- por la altura de mi mano cuando les de la señal. Ahora les daré una serie de señales con mi mano; y cada una de éstas representará una textura sonora diferente. (Schafer, 1965, p. 35)

Los estudios más representativos en este campo son los realizados por Susana Espinosa, en los cuales incide en la importancia de crear situaciones de enseñanza-aprendizaje que estimulen el impulso creativo, la improvisación y la exploración de nuevos códigos. Así mismo, define la creación sonora en tiempo real como una experiencia participativa que posee cualidades excepcionales para alcanzar resultados musicales satisfactorios e inmediatos con grupos de personas músicos o no músicos (Espinosa, 2005).

Coincidimos con Espinosa en que los aspectos básicos que contiene la creación sonora en tiempo real están relacionados con eliminar de las aulas algunos elementos restrictivos tradicionales. Nuestro objetivo al analizar el uso de Soundpainting en el ámbito educativo se relaciona también con esta posición.

\subsection{Estudios actuales sobre Soundpainting}

El estado de la cuestión sobre estudios relacionados con el tema de investigación a nivel general, dos interesantes trabajos sobre Soundpainting nos revelan el interés creciente que este lenguaje está teniendo entre los investigadores:

En su tesis doctoral, Soundpainting as a system for the collaborative creation of music in performance, Duby (2006) se plantea la siguiente pregunta de investigación: ¿Cómo funciona Soundpainting como sistema para la creación colaborativa en la interpretación musical? Destacamos aquí dos aspectos importantes encontrados en las conclusiones de este trabajo: (i) que Soundpainting ofrece a los músicos en cualquier nivel formativo (esto es importante y se relaciona con nuestra investigación) un espacio para la exploración de aspectos interpretativos (básicos y avanzados); tiene potencial de método, sobre todo para la creación a diferentes niveles; alumno-profesor, y (ii) la dimensión lúdica que posee este len- 
guaje, la cual, además de favorecer la creatividad, fomenta la comunicación y la colaboración en el proceso creativo.

En otro sentido, el trabajo de Helen Julia Minors publicado en Les Cahiers de la Société québécoise de recherche en musique bajo el título Music and Movement in Dialogue: Exploring Gesture in Soundpainting (Minors, 2012) encuentra hallazgos relacionados con la dialéctica existente entre música y movimiento. Soundpainting es tratado como estudio de caso en el cual se examina cómo la música y la danza pueden crear y contribuir a un diálogo entre las artes, cuyo objetivo es ilustrar cómo se forman los diálogos entre música y movimiento en este lenguaje. Concluye que tanto el lenguaje como el proceso creativo se basan en una premisa: que tanto los músicos como los bailarines, y tanto los coreógrafos como los compositores comparten un entendimiento gestual, y por tanto son capaces de compartir el lenguaje.

\subsection{Soundpainting en el entorno educativo}

El propósito de esta investigación ha sido analizar cómo Soundpainting se ha usado y se está usando en diferentes ámbitos educativos. Para ello, fueron revisados y analizados dos estudios, ya que observamos en ellos cierta relación con nuestro objeto de estudio y con nuestros objetivos de investigación.

El estudio de Puigdemasa (2012), aunque realiza una investigación específica del lenguaje Soundpainting centrada en la educación secundaria, no lo aborda desde el punto de vista de la composición en tiempo real. Analiza cómo puede ser usado como estrategia de aprendizaje para desarrollar la inteligencia emocional en los alumnos. Describe un conjunto de actividades basadas en la creación de una orquesta de improvisadores concluyendo que el profesorado entrevistado considera que la propuesta reúne las características y ofrece las herramientas pertinentes para poder conseguir los objetivos que se propone.

O ensino musical num centro rural galego (Secundária) es un artículo relacionado con el uso de Soundpainting en el ámbito educativo, realizado por Rodrigues (2012). Basado en la experiencia del autor en aplicar Soundpainting como experiencia didáctica en el aula, se plantea si se puede utilizar como recurso creativo para alumnos sin conocimientos musicales. Concluye que "A finalidade pode ser muita dependendo dos gestos que se usem". Observando los resultados, estamos de acuerdo con tal afirmación. También coincidimos con el autor cuando dice que, lo importante no es el producto final de la obra, sino el proceso en el que cada intérprete suena libremente en función de unas reglas básicas preestablecidas (Rodrigues, 2012, p.40).

\section{Metodología de la investigación}

Tal y como define Ibarretxe (2006) la metodología de la investigación, planteamos nuestra investigación desde el enfoque del paradigma interpretativo, ya que a partir del análisis hemos querido comprender e interpretar los resultados. Asumimos pues la metodología cualitativa que ha justificado la elección del método o diseño de la investigación, así como las técnicas e instrumentos de recogida de datos. 


\subsection{Diseño de la investigación}

El diseño de esta investigación se presentó como un estudio de caso colectivo (Stake, 1994) realizado en tres ámbitos educativos diferentes. La calidad y credibilidad de la muestra se basó en la alta cualificación que poseen los tres profesores respecto de nuestro objeto de estudio, tal y como lo demuestran sus diplomas oficiales en el segundo nivel de Soundpainting. Además se comprobó que los tres tienen una amplia experiencia en la aplicación del lenguaje en diferentes ámbitos educativos, que era nuestro escenario de observación.

- Conservatorio de Privas (Rhône les Alpes, Francia)

- Escuela de Música de Tres Cantos (Madrid, España)

- Jossefiau Primary School (Salzburgo, Austria)

Cuando se definió el problema de la investigación, se delimitaron una serie de preguntas iniciales. Estas preguntas fueron reformuladas durante todo el proceso de investigación. Partimos de una serie de categorías teóricas iniciales que se complementaron con categorías emergentes surgidas de un primer análisis de todo el contenido. Con todas ellas se realizó un proceso de refinado antes de plantear el mapa definitivo de categorías que darían respuesta a las preguntas de la investigación.

La metodología aplicada consistió en diferentes técnicas cualitativas que incluyeron la observación participante, las notas de campo y las entrevistas. Tanto las entrevistas como las sesiones de trabajo quedaron registradas en vídeo.

El estudio se concentró en observar y analizar la aplicación de Soundpainting en el ámbito educativo desde un enfoque descriptivo (se fundamenta en estudios existentes) bajo un paradigma de investigación interpretativo con la finalidad de comprender y describir cómo se usa este lenguaje en diferentes ámbitos educativos, para lo cual se realizó una triangulación interna de la codificación de los datos cualitativos entre los tres agentes (Colás y Buendía, 1998).

\subsection{Técnicas e instrumentos de recogida de datos}

Las técnicas de recogida de datos usadas en este estudio se enmarcan dentro de la investigación cualitativa situándolas en el enfoque de las técnicas directas o interactivas (Colás y Buendía, 1998), ya que contemplan la presencia del investigador en el contexto de la investigación frente al informante.

Se realizó la observación en las sesiones de trabajo durante las cuales se tomaron notas de campo. Además, el proceso de enseñanza fue registrado en vídeo para su posterior análisis con el software ELAN de análisis cualitativo, con el fin de codificar y categorizar el contenido.

Se hizo una entrevista semiestructurada a cada uno de los profesores informantes, la cual fue validada por tres doctores expertos en la temática estudiada. Las entrevistas incluyeron ocho preguntas iniciales estructuradas en tres áreas temáticas: percepciones del profesor en cuanto a los resultados observados en la aplicación de Soundpainting, didáctica y estrategias metodológicas y finalidades en la aplicación del lenguaje. 
En el caso de la Escuela de Música de Tres Cantos, al finalizar la clase se invitó a un grupo de cinco alumnos a realizar una entrevista abierta estimulada por la reproducción en vídeo de la sesión de trabajo en la que habían participado. Durante la entrevista se intentó mantener un clima grupal abierto y flexible para propiciar las opiniones de los alumnos, tomando un enfoque no directivo. Solo en los momentos en los que los alumnos estuvieron menos participativos se introdujeron preguntas para estimular respuestas grupales.

\subsection{La muestra}

Los agentes implicados en esta investigación fueron tres centros educativos en los que se analizaron grupos de alumnos con sus respectivos profesores. Se realizó un muestreo por conveniencia y un muestreo intencional (Gutiérrez, 1999) de acuerdo con las técnicas de muestreo de la investigación cualitativa. El muestreo intencional se decidió según el nivel competencial en Soundpainting que tienen los tres profesores, después de haber observado que podían aportar información variada para la investigación, ya que su conocimiento sobre el tema a estudiar es profundo y cualificado; los tres docentes poseen el segundo nivel de Soundpainting.

\subsection{Procedimiento de análisis de los datos}

Después de transcribir y traducir en algunos casos las entrevistas, los archivos fueron importados al software de análisis de contenido ATLAS.TI como documentos primarios para su codificación y categorización. También se importaron los archivos resultantes de la codificación de las grabaciones en vídeo de las sesiones de trabajo haciendo uso del programa de software libre ELAN para análisis de contenido.

La codificación, tanto en ELAN como en ATLAS.TI, se hizo a partir de un listado inicial de categorías a las cuales se fueron añadiendo otras emergentes, siguiendo las directrices del análisis de contenido (Krippendorf, 1990), (Anguera, 1986) diferenciando entre unidades de contexto y unidades de registro. Dada la amplitud del mapa de categorías se dividió en categorías principales y subcategorías.

De la codificación abierta se pasó a una segunda fase de codificación selectiva (Strauss y Corbin, 1998) en la cual se sintetizaron y agruparon los datos haciendo un refinado de los códigos obtenidos para agruparlos por familias. Así pues, se hizo un análisis de todo el contenido para detectar las categorías previas y otras no contempladas (emergentes).

Tabla 1. Categorías y subcategorías iniciales de la investigación

\begin{tabular}{|l|l|}
\hline Categorías Iniciales & Subcategorías \\
\hline \multirow{4}{*}{ Ámbitos Educativos } & Educación Primaria \\
\cline { 2 - 2 } & Conservatorio \\
\cline { 2 - 2 } & Escuela de Música \\
\cline { 2 - 2 } & Niveles alumnos \\
\hline Aplicaciones didácticas Soundpainting & Gestos \\
\hline Resultados del uso de Soundpainting & Percepciones de los profesores \\
\hline
\end{tabular}




\begin{tabular}{|l|l|}
\hline \multirow{2}{*}{ Composición/Creación en tiempo real } & Individual \\
\cline { 2 - 2 } & Participación activa \\
\cline { 2 - 2 } & En grupo/Colectiva \\
\hline Cognición musical & Aprender el lenguaje \\
\hline \multirow{2}{*}{ Desarrollo de Capacidades Musicales } & Creatividad \\
\cline { 2 - 2 } & Expresión Musical \\
\cline { 2 - 2 } & Improvisación \\
\cline { 2 - 2 } & Atención y Escucha activas \\
\hline Desarrollo de otras Capacidades & Motivación \\
\hline
\end{tabular}

Para dar garantía a los datos obtenidos y credibilidad a nuestra investigación se utilizó la triangulación interna (Colás y Buendía, 1998 y Cohen y Manion, 1990) para contrastar la información, cruzando los datos obtenidos de los tres informantes que participaron en el estudio, buscando coincidencias y/o divergencias entre ellos. La triangulación de técnicas consistió en aplicar a nuestra investigación diferentes técnicas e instrumentos de recogida de datos como fueron la observación (video y notas de campo), las entrevistas que fueron validadas por expertos (primera triangulación de la investigación) de acuerdo con los criterios de adecuación y pertinencia, y el análisis de contenido (ELAN y ATLAS.TI). A este tipo de triangulación Janesick (1994) citado en Gutiérrez, (1999) la denomina triangulación de datos.

\section{Análisis y discusión de los resultados}

Una vez transcritas las entrevistas, se hizo una revisión de la observación en vídeo y de las notas de campo, para dar paso al proceso de codificación para categorizar los datos. Se hizo un análisis de los datos de cada centro educativo por separado (análisis de los datos) para después triangular los datos y pasar a la comparación de resultados entre ellos (discusión de resultados).

\subsection{Primer caso: Conservatorio de Privas}

Se realizaron varias visitas al Conservatorio de Privas en la región de Rhône les Alpes (Francia) en el mes de marzo de 2014. El conservatorio ofrece durante el curso escolar 2013/2014 un proyecto innovador sobre Soundpainting que está financiado por el Consejo General del Departamento de Ardèche. El proyecto tiene como objetivo introducir Soundpainting como formación creativa complementaria dentro del currículo del alumnado.

El grupo lo conformaban instrumentos de cuerda, de viento-madera (flautas), de viento-metal (trompetas y trombones), y contó con la ayuda de una profesora de refuerzo especializada en danza contemporánea. En actividades relacionadas con la creación en tiempo real, se dio la oportunidad a diferentes alumnos de componer sus propias piezas, en este caso a modo de creación colectiva, dado que todos los alumnos que quisieron fueron intérpretes y Soundpainters en diferentes momentos de la actividad. Aunque se observaron errores cuando los alumnos indicaban algunos gestos, los intérpretes continuaron tocando ( $1^{\mathrm{a}}$ norma de Soundpainting, el error no existe). En palabras del profesor extraídas de la entrevista: 
Con esta negación del error se motiva a los alumnos para ser creativos.

Los resultados de la entrevista revelaron diferentes estilos de aprendizaje que se produjeron al aplicar Soundpainting en el aula. Una de las percepciones del profesor cuando ha trabajado en el ámbito de la educación primaria fue que:

(...) para los niños es muy fácil de aprender, quiero decir, no tienes que explicar qué es Hit cuando ellos conocen Long Tone ¿Sabes? Es evidente: es lo contrario.

Se refiere a que el lenguaje Soundpainting es muy intuitivo, lo cual se traduce en un aprendizaje por descubrimiento tal y como se puede deducir del comentario anterior.

Se observó que en todas las actividades se facilitaba la motivación de los alumnos y se reforzaba su concepto de autoestima, lo cual les estimulaba a ser espontáneos en las respuestas aumentando así su capacidad creativa, musical y personal. El desarrollo, tanto de la atención como de la escucha activa, está íntimamente ligado a cualquier actividad grupal que precise de la comprensión de ciertos códigos (gestos) para poder ofrecer una respuesta inmediata. Así pues la aplicación del lenguaje Soundpainting estimuló el desarrollo de estas capacidades en los alumnos.

Entendemos pues, que esa relación entre juego y libertad propicia el desarrollo creativo. En ese sentido el profesor Nid comentó:

(...) es como un juego, un puzzle. Tú juntas las cosas y entonces puede ser que des la vuelta a la pieza y se convierta en otra. Son cosas libres, es muy asombroso por eso.

Las estrategias metodológicas aplicadas por el profesor Nid tuvieron como finalidad el aprendizaje del lenguaje Soundpainting, por una parte, y, por otra, crear situaciones para la creación en tiempo real. Sobre el uso de Soundpainting en el aula como herramienta para que los alumnos puedan componer, el profesor explicó que:

Yo busco lo más eficiente para permitir a los niños componer algo, es muy estructurado para ellos (...) y ellos hacen la composición. Por ejemplo, les permito usar Long Tone, Hits, Shapeline, en Launch Mode por ejemplo, y simplemente a hacer la pieza con ello. A veces un poco de Scanning, y hacen cosas hermosas.

Se observó que al hacer adaptaciones del lenguaje se estaba atendiendo a la diversidad del alumnado, fomentando así la participación activa dentro del grupo.

\subsection{Segundo caso: Escuela de Música de Tres Cantos}

Durante el mes de abril de 2014 se observaron varias clases colectivas de oboe en la Escuela de Música de Tres Cantos (Madrid, España) con un profesor y un grupo de alumnos con diferentes niveles.

Las estrategias didácticas del profesor combinaron momentos de improvisación con otros específicos de expresión musical, en los que se trabajaron aspectos específicos del instrumento. Se observó que el uso de ciertos gestos de Soundpainting se fundamentaba en sus finalidades. 
En algunas de las actividades se observó que el profesor aprovechó tanto los errores de algún alumno como intervenciones no deseadas para crear pequeñas piezas musicales en tiempo real. Además facilitó la motivación quitando importancia al hecho de que los dos alumnos le hubiesen interrumpido. La percepción de los alumnos, expresada durante la entrevista grupal, fue que:

Alumno 4: "Ha quitado la tensión a la explicación, estábamos todos atentos a la explicación... Ellos han empezado a hablar y él lo ha escuchado y ha aprovechado el momento"

Alumno 3: "Ha pasado una cosa extraña y ha aprovechado que... digamos para sacarle partido, ha visto que mi compañero me ha preguntado por la camiseta y ha dicho voy a sacarle partido, voy a caricaturizarlo un poco, y esto a la hora sobre todo de hacer una creación con Soundpainting le da mucha gracia"

Además de facilitar la motivación de sus alumnos, le sirvió en algunos momentos como hilo conductor de la clase y en otros para crear situaciones en las que se realizaron composiciones en tiempo real. Sus palabras al respecto fueron:

El profesor tiene que estar rápido y ver que si ha hecho una cosa que no era... pero bueno, pues ahora... ahora te engancho por esta. Sigue, sigue, sigue, y eso tiene... como energía de verdad, es energía. De ahí lo saca y al resto les parece interesante.

La aplicación didáctica del lenguaje Soundpainting que realiza en sus clases colectivas, responde a un interés por ensanchar las posibilidades expresivas del oboe, dado que, como nos comentó en la entrevista, se trata de un instrumento limitado en ese sentido:

También es cierto que los instrumentos están construidos para que suenen dentro de un ámbito de "lo agradable", y entonces si te sales de ahí ya no está bien, si te vas para acá tampoco está bien, y como con el Soundpainting lo importante, digamos que la forma adquiere significado por la intención con la que lo haces, eso desaparece pero curiosamente aparece mejor calidad sonora que cuando tocan siguiendo una partitura, o por lo menos más viva.

La escucha activa se observó tanto en el desarrollo de capacidades musicales de los alumnos como en el desarrollo personal. En lo que se refiere a los procesos donde se usó Soundpainting para la creación en tiempo real, la percepción del profesor extraída de la entrevista fue:

Entonces, están atentos, porque todas las indicaciones se hacen en un momento y no hay nada donde puedas mirar y volver a repetir, la atención viene ahí muy rápido, y luego el tema de responder en el momento también eso es... es lo más representativo.

Respecto a la respuesta en el momento, se observó que los alumnos fueron siempre espontáneos, tanto en las respuestas a los gestos de Soundpainting como en lo referente a su personalidad. En ningún caso se observó que los alumnos desarrolla- 
sen actitudes de timidez o vergüenza hacia el hecho creativo. La actitud creativa del profesor pudo ser un factor determinante, tomando en cuenta sus propias palabras:

No hay una forma exacta de qué tiene que ser el puntillismo (ritmo irregular en el que se combinan sonidos largos y cortos interpretados rápidamente), sino que es muy amplio, o un sonido largo, que puede ser de muchos sonidos y de distintas texturas, timbres, todo... pues también, pues si uno es tímido puede hacerlo tímido no pasa nada, yo puedo trabajar con él, para que sea... subirlo, bajarlo... entonces la participación del alumnado es en ese sentido mejor.

En algunos momentos el profesor empleó estrategias metodológicas relacionadas con el uso didáctico de los gestos de Soundpainting. Se utiliza aquí el término "uso didáctico" del gesto para diferenciarlo del uso que tiene dentro del lenguaje; estructurar la composición y ofrecer material sonoro, visual o de movimiento al Soundpainter. Entendemos, pues, que cuando un gesto es sacado del contexto sintáctico del lenguaje y es usado para desarrollar ciertas capacidades en los alumnos, se está haciendo un uso didáctico del mismo.

Otro aspecto metodológico que se observó fue el uso lúdico que el profesor hacía del lenguaje en distintos momentos de las sesiones colectivas. En el siguiente comentario se observa una relación entre atención y juego, la cual favorece el interés por aprender de los alumnos, tal y como nos explicó en la entrevista:

(...) les incentiva mucho, les estimula a nivel intelectual, la atención se la capta rápido, porque es un juego, cuando hablamos de niños ya es un juego, la idea de juego eso ya anima, la idea de obligación delante de una partitura pues, eso ya cansa un poco.

\subsection{Tercer caso: Jossefiau Primary School, Salzburg.}

En la Josefiau Primary School de Salzburgo (Austria) se observó a un grupo de alumnos de integración con NEE (necesidades educativas especiales) y a su profesora.

El grupo de alumnos estaba formado por diez niños y tres niñas entre los que se encontraban un alumno con síndrome de Down, tres alumnos con TDH y otros con deficiencias en su nivel de aprendizaje, según comentó su profesora en la entrevista.

Estas clases pertenecen a un proyecto que la escuela ha contratado con el Toihaus Theater de Salzburgo. El proyecto cuenta con la ayuda de dos profesoras de refuerzo: una de ellas es músico y la otra bailarina.

Uno de los aspectos más interesantes observados en las clases de la profesora fue, tal y como ella comentó en la entrevista posterior, que se convertía en un personaje para interactuar mejor con sus alumnos y que ellos la sientan más cercana, haciendo un uso lúdico del lenguaje Soundpainting. En esos momentos ella no era su profesora, era más una animadora:

No estoy como una profesora. Si son más pequeños soy una amiga de juegos, si son más grandes, digamos que una entrenadora de artes, una improvisadora o algo así. Este juego creo que es la finalidad más grande. 
Como nos indicó en la entrevista posterior, su estrategia didáctica con Soundpainting era hablar lo menos posible a los alumnos para provocar un aprendizaje por descubrimiento. Para ello, su estrategia didáctica se basó en el aprendizaje por imitación: primero lo realizaba con alguna de las profesoras de apoyo y luego lo pedía a toda la clase; casi siempre sin explicación del gesto, es decir, sin contenidos teóricos y desde la práctica.

En otros momentos de la sesión surgieron preguntas y curiosidades por parte de los alumnos sobre cómo realizar ciertos contenidos sonoros, es decir, de manera natural emergió un interés por aprender el lenguaje por parte de los niños. A partir de ese momento, la profesora invitó a los alumnos a participar en la creación de algunas frases Soundpainting.

Una de sus finalidades en la aplicación de Soundpainting en este grupo fue desarrollar ciertas capacidades en sus alumnos. Ella nos comentó que aunque se producen burlas y mofas entre ellos, el hecho de que el resultado sea colectivo les hace sentirse responsables y apoyarse unos a otros, como en este caso:

(...) también les está dando una situación de alivio (bienestar) a los niños. Se burlan los unos de los otros, cuando uno se equivoca todo el mundo se ríe, y dicen no deberías hacer eso, ahora deberías estar en silencio. Pero no es para mofarse o hacerle sentir triste, ellos están corrigiendo para que todo el grupo (es lo que yo pienso), para que todo el grupo pueda hacer algo bonito, por ejemplo. Este es el resultado que, quiero decir, el resultado que últimamente estoy observando en mis clases.

El desarrollo de la escucha y la concentración fue un dato importante aparecido en diferentes momentos de la entrevista con la señora Oran. Tal y como nos comentó, el participar en una actividad de composición grupal como Soundpainting requiere que ciertos parámetros entren en funcionamiento sin que los alumnos se den cuenta:

(...) ellos también quieren prestar atención, quieren aprender, pero de cualquier manera el Soundpainting en sí mismo necesita mucha atención. Quiero decir, tú no has de tener 3 o 10 años... Cuando estamos tocando para los demás, tú tienes que estar muy atento y muy concentrado para observar lo que está ocurriendo, lo que está señalando el Soundpainter, lo que está pasando en el grupo. Así que hay muchos parámetros actualmente sin querer indicarlos y los niños también sin pensar en ello tienen que concentrarse en muchas cosas y yo creo que eso les está dando un desarrollo.

Un dato destacable observado en las respuestas de la entrevista fue que la integración de alumnos poco sociables en el grupo se daba en sus clases cuando usaba Soundpainting, en parte por la motivación que la profesora les daba. Así pues al desarrollarse esta capacidad de socialización en el alumnado, directamente se estaba reforzando su autoestima:

Con los niños es un poco como hacer brillar su carácter, siempre lo observo esto. Y fue también cuando estaba enseñando el pasado año por ejemplo en una escuela, había algunos profesores que me daban feedback sobre los niños y me hablaron sobre un muchacho (lo recuerdo porque fue muy satisfactorio usando el lenguaje 
Soundpainting) y después de la clase me dijeron que ese muchacho era muy callado, no sociable, no se comunicaba mucho, pero cuando yo pregunté después de terminar la clase quién quería hacer Soundpainting él fue el primero en decir yo. Esto fue una herramienta para romper sus barreras.

En lo referente a capacidades musicales de los alumnos, la profesora comentó que una de sus percepciones era que se desarrollaba la creatividad de sus alumnos cuando respondían a los gestos que ella les signaba:

Yo creo que mientras ellos responden a las cosas, mientras que responden a los signos ven las posibilidades y saltan al próximo nivel, cada vez que están interpretando conmigo, básicamente. Y esta es la idea de abrir su creatividad.

Se observaron diferentes tipos de aprendizaje en la clase de la profesora. En cuanto al aprendizaje cooperativo, la profesora comentó que los alumnos se sentían igual de cómodos siendo líderes cuando componían que siendo parte del grupo cuando eran intérpretes:

Es una herramienta que iguala a cada niño. Porque es un sistema muy claro, una persona pide (con gestos) y el resto del grupo responde, da igual las habilidades o lo bueno que seas aquí (en la clase), lo alto que seas, lo pequeño... Mientras puedas mover tus brazos, vas a tener tu momento de líder y el resto del grupo te va a responder. Es como un tipo de... Como una herramienta de igualdad para mí.

También se observó que se desarrollaba el aprendizaje autónomo cuando la profesora nos comentó que sus alumnos se interesaban por el lenguaje de forma natural, sin ser forzados a aprenderlo. Y contó una anécdota interesante sobre la sesión observada anterior a la entrevista:

Entonces ellos vienen y te dicen: podemos hacer esto como esto, o puedo signar ahí y entonces cuando ellos vienen a hacer Soundpainting tú también ves cómo ellos están construyendo y cómo ellos están usando el lenguaje para sí mismos. Esto según su creatividad, ahí no puedo hacer nada. Ayer, un niño, uno de los niños combinaba la voz abriendo y cerrando con risa por ejemplo y eso no se lo enseñé.

Tanto en la entrevista como en la observación y las notas de campo se observó que la profesora hizo participar en todo momento a sus alumnos en el proceso de la creación en tiempo real, fomentando así la participación activa, dejando que cada uno de sus alumnos participase activamente como líder o como integrante del grupo. En la entrevista hizo la siguiente reflexión:

Hasta ahora yo nunca he visto ningún niño que no haya querido participar, por ejemplo. Había este niño que estaba sentado así todo el tiempo (ayer) y yo le dije así no vas a poder ver los signos y entonces él se puso así, él tuvo que mirarme (...). 


\subsection{Discusión de Resultados}

Después de haber analizado los datos obtenidos de la observación realizada durante las visitas a los tres centros educativos implicados en el estudio, así como de las entrevistas realizadas a cada uno de los profesores, se hace necesaria una discusión de los resultados con el objetivo de triangular los datos. El proceso de discusión se fundamenta en nuestro marco teórico y en las categorías que de él se extrajeron, así como en las que emergieron durante el proceso de categorización de los datos.

Tabla 2. Categorías y subcategorías iniciales de la investigación

\begin{tabular}{|l|l|}
\hline Categorías Iniciales & Subcategorías \\
\hline \multirow{4}{*}{ Ámbitos Educativos } & Educación Primaria \\
\cline { 2 - 2 } & Conservatorio \\
\cline { 2 - 2 } & Escuela de Música \\
\cline { 2 - 2 } & Niveles alumnos \\
\hline Aplicaciones didácticas Soundpainting & Gestos \\
\hline Resultados del uso de Soundpainting & Percepciones de los profesores \\
\hline \multirow{4}{*}{ Composición/Creación en tiempo real } & Individual \\
\cline { 2 - 2 } & Participación activa \\
\cline { 2 - 2 } & En grupo/Colectiva \\
\hline Cognición musical & Aprender el lenguaje \\
\hline \multirow{4}{*}{ Desarrollo de Capacidades Musicales } & Creatividad \\
\cline { 2 - 2 } & Expresión Musical \\
\cline { 2 - 2 } & Improvisación \\
\cline { 2 - 2 } & Atención y Escucha activas \\
\hline Desarrollo de otras Capacidades & Motivación \\
\hline
\end{tabular}

Categorías y subcategorías emergentes de la investigación

\begin{tabular}{|l|l|}
\hline Categorías Emergentes & Subcategorías \\
\hline \multirow{4}{*}{ Desarrollo de Capacidades Musicales } & Expresión Corporal \\
\cline { 2 - 2 } & Análisis Musical \\
\hline \multirow{4}{*}{ Estilos de Aprendizaje } & Espontaneidad \\
\cline { 2 - 2 } & Creatividad \\
\cline { 2 - 2 } & Refuerzo de la Autoestima \\
\hline & Autónomo \\
\cline { 2 - 2 } & Cooperativo \\
\cline { 2 - 2 } & Por descubrimiento \\
\cline { 2 - 2 } & Por error \\
\cline { 2 - 2 } & Imitativo \\
\hline \multirow{2}{*}{ Composición/Creación en tiempo real } & Estilos musicales \\
\cline { 2 - 2 } & Procesos \\
\hline
\end{tabular}




\begin{tabular}{|l|l|}
\hline \multirow{4}{*}{ Estrategias Metodológicas } & Warm ups \\
\cline { 2 - 2 } & Uso didáctico de los gestos \\
\cline { 2 - 2 } & Uso lúdico del lenguaje \\
\cline { 2 - 2 } & Adaptación del lenguaje por niveles \\
\hline
\end{tabular}

El objetivo de nuestra investigación planteaba dos dimensiones claras: analizar el uso que se está haciendo en el ámbito educativo del lenguaje Soundpainting y conocer con qué finalidad se aplica. Así pues, derivadas de este objetivo, nos planteamos tres preguntas de investigación.

\section{¿Qué diferencias o similitudes existen en la aplicación didáctica de Soun- dpainting en los diferentes ámbitos educativos observados?}

En los tres casos estudiados se ha aplicado el lenguaje Soundpainting desde dos perspectivas o procesos. Una de estas dos aplicaciones consistió en usar los gestos del lenguaje fuera de su contexto, es decir, no para la creación en tiempo real y sí como estrategia didáctica para obtener diferentes metas. Los contenidos de los gestos de Soundpainting permiten elaborar diferentes actividades que fuera del proceso de creación ayudan a desarrollar diversas capacidades en los alumnos. Así pues, esta similitud en la aplicación didáctica de los gestos extraídos de su contexto, ha sido una constante general en cada centro educativo.

Desde la perspectiva de la creación en el aula es donde existen diferencias en la aplicación del lenguaje. En el uso de la herramienta que hace el profesor es donde encontramos que dependiendo de la finalidad, la usa en una u otra dirección.

Las diferencias que se dieron en la escuela de primaria fueron que la profesora priorizó el desarrollo de capacidades en los alumnos sobre los procesos de creación, aunque siempre propició situaciones en las que los alumnos pudieron salir a componer sus propias piezas.

En la clase colectiva de la Escuela de Música, la aplicación de Soundpainting estuvo dirigida a trabajar la capacidad expresiva de los alumnos con su instrumento. Este caso difiere de los otros dos en las metas, pero no en las estrategias adoptadas por el profesor.

La aplicación didáctica de Soundpainting se ha dado en los tres casos desde la perspectiva del juego, haciendo un uso lúdico del lenguaje lo cual propicia la participación activa del alumnado, eligiendo ellos mismos el rol que quieren desempeñar en cada momento; ya sea como líder (animador) o como integrante.

Soundpainting facilita que, incluso siendo parte del grupo de intérpretes, los alumnos puedan escoger diferentes roles en cada momento, ya que algunos de los gestos permiten diferentes actitudes en diferentes momentos de la composición. Es pues una herramienta de aplicación didáctica que permite tanto el desarrollo individual dentro del colectivo como la colaboración con los demás integrantes. Con Soundpainting los alumnos están tomando decisiones en todo momento, ya sean musicales (respuestas sonoras a los gestos) o en la elección del papel que quieran desempeñar dentro del grupo. Además, es un lenguaje que permite su adaptación dependiendo de los niveles educativos en los que se esté usando. 


\section{¿Qué tipo de capacidades se desarrollan en la aplicación didáctica de Soundpainting? ¿De qué manera se desarrollan?}

Diferentes capacidades, que han sido agrupadas en dos categorías, se han desarrollado al aplicar Soundpainting: capacidades musicales y capacidades personales (o de actitudes). De todas ellas destaca el desarrollo de la creatividad no sólo desde la dimensión musical sino también desde el desarrollo personal del alumno.

Desde la perspectiva musical, la creatividad en Soundpainting se da desde dos procesos bien diferenciados pero que en ambos se ha desarrollado esta capacidad; cuando el niño es el compositor de la obra y cuando ha de responder a los gestos que el profesor u otro alumno le esté signando. Parece ser que la participación activa de los alumnos facilitó este desarrollo al igual que la adaptación del lenguaje que los profesores hicieron por niveles. Así pues, facilitando la participación activa de los alumnos, tanto la interpretación como la creación musicales en Soundpainting ayudan a desarrollar la capacidad creativa.

La capacidad de escucha activa es otro aspecto destacable en la aplicación del lenguaje. Como en cualquier juego de niños, si queremos participar primero deberemos aprender las reglas del juego. Soundpainting, como sistema para la creación en tiempo real posee estas reglas de juego que deben ser aprendidas si se quiere participar. Así pues, haciendo un uso lúdico del lenguaje, la participación de los alumnos requiere de una escucha y atención constantes, dado que las reglas no sólo se dan en los procesos iniciales de aprendizaje del lenguaje sino también durante el proceso de preparación y creación sonora. Es pues en este proceso de la creación en tiempo real donde la escucha se torna más activa, ya que el proceso requiere del alumno una atención constante a todo el hecho sonoro y visual que está ocurriendo en el momento. Es un bucle que sigue el orden: escucha-acción-reacción.

La expresión corporal y musical han sido dos aspectos en los cuales los profesores insistieron para lograr una mejor calidad sonora en las interpretaciones de sus alumnos. La expresión corporal ha servido en muchos momentos como medio para alcanzar el fin de la expresión musical. La aplicación de algunos gestos del lenguaje Soundpainting como estrategia didáctica permite que los alumnos experimenten en primer lugar con sus cuerpos, para después expresar el contenido sonoro del gesto de forma musical.

El desarrollo de capacidades personales como la motivación y el refuerzo de la autoestima, son producto de una atención a la diversidad en el aula que se ha dado mediante la adaptación de los gestos del lenguaje Soundpainting por niveles intelectuales.

\section{Conclusiones}

Esta investigación nos ha proveído de datos interesantes en gran parte por la decisión de haber realizado un estudio de casos múltiple, lo cual nos permitió triangular los datos centrándonos en su visión del objeto de estudio y a la vez comprobarlos con diversas fuentes. Además de la triangulación de fuentes, se realizó una triangulación de métodos para validar y dar garantías de calidad a la investigación.

Después de realizar una revisión documental para conocer el estado de la cuestión se encontraron diversos hallazgos referentes a estudios relacionados con nuestro 
objeto de estudio, así como información importante para concretar el marco metodológico de la investigación.

La primera conclusión a la que llegamos se dio en la fase de selección de la muestra, cuando pudimos obtener tres casos en tres ámbitos educativos diferentes y a la vez variados: un conservatorio de música, una clase colectiva instrumental en una escuela de música, con un grupo de alumnos con necesidades educativas especiales en un centro de educación primaria. Así pues, tenemos datos (aunque no generalizables) que confirman la paulatina introducción del lenguaje Soundpainting en estos centros educativos.

Soundpainting se ha aplicado en el aula utilizando diferentes estrategias metodológicas activas en las cuales la participación activa del alumnado ha sido una constante. Detectamos que estas situaciones se han dado en forma de atención a la diversidad y promoviendo actividades desde la perspectiva del juego. Así pues, concluimos que la aplicación de Soundpainting en el aula permite que los alumnos escojan diferentes roles de participación entre los que podemos encontrar el de líder, cuando es compositor; o el de formar parte del grupo cuando decide ser intérprete. En los dos casos, las aportaciones individuales de los alumnos han formado parte de un trabajo colectivo que se ha traducido en una forma de aprendizaje cooperativo. Podemos afirmar que la aplicación de Soundpainting desde la perspectiva del juego ha facilitado la motivación de los alumnos y ha reforzado su autoestima, desarrollando así sus capacidades personales.

Coincidimos con estudios similares en los que se identifica al profesor con el rol de animador. En ese sentido creemos oportuno citar a Espinosa (2005, p. 33): "El animador trabaja con un sentido de "juego", entendiendo por ello aquella acción en equipo donde cada uno cumple su rol pero teniendo en cuenta el rol del otro (...)"

Los gestos del lenguaje Soundpainting se han usado desde dos procesos de enseñanza diferentes. En un primer proceso, los gestos se enseñan desde el aprendizaje imitativo o por reiteración para luego ser usados con la finalidad de crear composiciones en tiempo real. Se puede concluir que este proceso trata el gesto como herramienta para alcanzar un fin artístico y de comunicación. En cambio, el otro proceso está basado en el uso didáctico del gesto para alcanzar metas diferentes a las de la composición en tiempo real. Asimismo, los gestos permiten su adaptación por niveles, tal y como ha quedado demostrado en los resultados; cada profesor diseñó un set de gestos adaptados al nivel de sus alumnos y a sus metas u objetivos.

En relación al uso didáctico de los gestos del lenguaje se detectó que facilita la expresión corporal y musical en los alumnos, creando situaciones de exploración y experimentación tanto del cuerpo como del instrumento. Entendemos que esta es la base para desarrollar la capacidad creativa de los alumnos y que la aplicación de Soundpainting en el aula les permite desarrollar dicha capacidad, tanto desde el punto de vista musical como personal.

Concluimos finalmente, que cualquier alumno, independientemente de su nivel intelectual o académico, participó del hecho creativo, bien como creador o como intérprete. Así mismo, el desarrollo de la capacidad creativa también quedó reflejado desde el punto de vista actitudinal en la espontaneidad que demostraron en sus respuestas a los gestos. 


\section{Líneas de investigación futuras}

Algunas investigaciones relacionan el uso de la música en la educación especial con resultados positivos en el desarrollo de competencias sociales y comunicativas (Darrow, 2006; y Moog, 1979; citados en Prause-Weber, 2006) como un beneficio secundario, es decir, cuando la música ha sido tratada como fin en sí misma. Sin embargo, sugieren que la música puede ser utilizada con una finalidad no musical, como un medio para un fin (Prause-Weber, 2006), explicando que en este caso se trataría de reforzar en los niños con necesidades educativas especiales las destrezas comunicativas o sociales, las destrezas motoras y la capacidad de atención (Moog, 1979).

Durante las visitas a las sesiones que la profesora realizó en la Jossefiau Primary School de Salzburgo con el grupo de alumnos con necesidades educativas especiales pudimos constatar que estaba usando Soundpainting en ese sentido. No como un fin en sí mismo sino como un medio para un fin: desarrollar en sus alumnos capacidades como la socialización, la autoestima o la capacidad de atención.

Lo que proponemos son investigaciones futuras en las que se planteen hipótesis o se realicen preguntas de investigación en relación a la aplicación de Soundpainting en la educación especial con el fin de encontrar y compartir ideas que ayuden a estos niños. En este sentido, encontramos un referente claro en las palabras de Prause-Weber (2006) cuando afirma:

Los profesionales se están esforzando en intercambiar y compartir sus conocimientos e ideas, con el fin de encontrar formas creativas de ayudar a los niños con necesidades especiales a través de la música y de permitirles participar de esta forma artística.

Soundpainting es un lenguaje que demanda de los alumnos en todo momento la experimentación sonora y visual. Así pues, la curiosidad o el descubrimiento, son formas de aprendizaje que se producen al usarlo en el aula, por lo que proponemos otra línea de investigación que plantee interrogantes o hipótesis de partida relacionados con el tipo de aprendizaje que se desarrolla en el aula al aplicar este lenguaje.

En relación a las dos propuestas expuestas anteriormente, uno de los hallazgos que se encontró fue que cada profesor tenía un set de gestos, a modo de caja de herramientas, los cuales usaba y adaptaba dependiendo del nivel de los alumnos. Así pues nos planteamos la siguiente pregunta ¿Existen gestos del lenguaje Soundpainting que desarrollen más que otros las capacidades de los alumnos?

\section{Referencias bibliográficas}

Alsina, P.; Díaz, M.; Giráldez, A.; Ibarretxe, G. (2009). 10 ideas clave. El aprendizaje creativo. Barcelona: Graó.

Anguera, M. T. (1986). La investigación cualitativa. Educar, 10. Recuperado de https://ddd. uab.cat/record $/ 35740$

Cárdenas, M.I. (2003). Evolución de la educación musical. La pedagogía de creación musical. Lugo: Unicopia

Cohen, L. y Manion, L. (1990). Métodos de investigación educativa. Madrid: La Muralla. 
Colas, M.P. y Buendia, M. (1998). Investigación educativa. Sevilla: Alfar.

Dessen, M. (2010). New Polyphonies: Score Streams, Improvisation and Telepresence. Leonardo Music Journal, 20. Recuperado de http://mdessen.com/wp-content/ uploads/2013/05/LEOMJ20_pp021-024.pdf

Díaz, M. (2006). Proceso de investigación. En M. Díaz (Coord.), Introducción a la Investigación en Educación Musical (102-116). Madrid: Enclave Creativa Ediciones.

Duby, M. (2006). Soundpainting as a system for the collaborative creation of music in performance. (Tesis doctoral, University of Pretoria). Recuperada de http://hdl.handle. net/2263/26959

Espinosa, S. (2005). Creación sonora en tiempo real. Una propuesta colectiva en la escuela secundaria. Aula de Innovación Educativa, 14(145), 28-33.

Gutiérrez, J. (1999). El proceso de investigación cualitativa desde el enfoque interpretativo y de la investigación-acción. Modelos de Análisis de la Investigación Educativa (9-59). Sevilla: Alfar.

Ibarretxe, G. (2006). El conocimiento científico en investigación musical. En M. Díaz, (Coord.), Introducción a la investigación en Educación Musical (8-30). Madrid: Enclave Creativa Ediciones.

Krippendorf, K. (1990). Metodología de análisis de contenido. Teoría y práctica. Madrid: Paidos.

Minors, H. J. (2012). Music and Movement in Dialogue: Exploring Gesture in Soundpainting. Les Cahiers de la Société québécoise de recherche en musique, 13(1-2), 87-96. doi: $10.7202 / 1012354 \mathrm{ar}$

Molina, E. (2008). La Improvisación: Definiciones y puntos de vista. Música y Educación, 75(3), 76-93.

Norro, M. (2006). Una exploración sobre la composición en tiempo real como intuición de la duración. Sonido, imagen y movimiento en la experiencia musical. Actas de la V Reunión de SACCoM, 109-125.

Prause-Weber, M. (2006): Tratamiento de la Música en alumnos con necesidades educativas especiales, Eufonía. Didáctica de la Música, 37. Barcelona: Graó.

Puigdemasa, A. (2012). Estrategias de enseñanza y aprendizaje musical y para el desarrollo de la inteligencia emocional en secundaria: improvisación con Soundpainting. (TFM, Universidad Internacional de La Rioja, Lleida). Recuperada de http://reunir.unir.net/ handle/123456789/763

Rodrigues, G. (2012). O ensino musical num centro rural galego (Secundária). Musicrearte digital, 1(1), 36-42. Recuperado de http://revista.musicrearte.com/musicrearte_digital_ numero01_2012_files/musicrearte_digital_01_2012.pdf

Rusinek, G. (2005). La composición en el aula de secundaria. Musiker, 14, 191-208. Recuperado de http://www.euskomedia.org/PDFAnlt/musiker/14/14191208.pdf

Schafer, R. M. (1965). El compositor en el aula. Buenos Aires: Ricordi Americana.

Smulovitz, S. [StefanSmulovitz ] (2010, March 21). The Mad Scientist Machine. [Archivo de vídeo] Recuperado de https://www.youtube.com/watch?v=VaueU35vwBM

Solís, H. (2006). Understanding Collective Gestural Improvisations; a Computational Approach. (Diploma de Estudios Avanzados, Universitat Pompeu Fabra, Barcelona). Recuperado de http://mtg.upf.edu/node/2219

Stake, R.E. (1994). Investigación con estudio de casos. Madrid: Morata.

Stanley, T. T. (2009). Butch Morris and the art of conduction. (Tesis doctoral, University of Maryland). Recuperado de http://hdl.handle.net/1903/9935 
Strauss, A., \& Corbin, J. (1998). Basics of qualitative research: Techniques and procedures for developing grounded theory. Thousand Oaks, CA: Sage.

Thompson, W. (2006). Soundpainting. The art of live composition. Workbook 1. New York: Thompson, W.

Zorn, J. (1984). Cobra. The game pieces. Recuperado de https://www2.ak.tu-berlin.de/ gastprof/collins/experimental-music/Zorn/americanmusic.28.1.0044.pdf 
